\title{
Influencia De La Adición De Cobre En La Corrosión Del Cromado Metálico Para Aplicaciones En La Industria Automotríz
}

\author{
Mario Alcides Lara Núñez. \\ Docente - Departamento de Energía y Mecánica \\ ESCUELA SUPERIOR POLITÉCNICA DEL EJÉRCITO - ECUADOR. \\ Segundo Manuel Espín Lago \\ Gonzalo Eduardo López Villacis \\ Jorge Patricio Guamanquispe Toasa \\ Docentes - Facultad de Ingeniería Civil y Mecánica \\ UNIVERSIDAD TÉCNICA DE AMBATO - ECUADOR. \\ Jorge Isaías Caicedo Reyes \\ Javier Enrique Orna Chávez \\ Docentes - Facultad de Mecánica
}

ESCUELA SUPERIOR POLITÉCNICA DE CHIMBORAZO- ECUADOR.

doi: 10.19044/esj.2017.v13n15p34 URL:http://dx.doi.org/10.19044/esj.2017.v13n15p34

\begin{abstract}
At present the use of metal coatings in the automotive industry is a priority so the present research had the objective of conducting studies of metallic chromium on surfaces of rings for buses applying national and international standards. The parameters of the coating process (time, temperature and voltage) are varied to check its incidence in corrosion by checking properties such as adhesion, hardness and layer thickness. It was determined that the optimum parameters for a nickel-chromium process are $60 \mathrm{~min}, 40^{\circ} \mathrm{C}$ and $7 \mathrm{~V}$, but with low corrosion resistance, so that an alternate coating was developed to add an alkaline copper layer, obtaining three layers, copper- nickel-chromium, thereby increasing corrosion resistance.
\end{abstract}

Keywords: Metal chromed, corrosion resistance, adherence, buses rings, alkaline copper layer

\section{Resumen}

En la actualidad el uso de recubrimientos metálicos en la industria automotriz es prioritaria por lo que la presente investigación tuvo por objetivo realizar estudios del cromado metálico en superficies de aros para 
buses aplicando normas nacionales e internacionales. Se varían parámetros del proceso de recubrimiento (tiempo, temperatura y voltaje) para comprobar su incidencia en la corrosión a través de verificar propiedades como la adherencia, la dureza y el espesor de capa. Se determinó que los parámetros óptimos para un proceso de níquel-cromo son de $60 \mathrm{~min}, 40^{\circ} \mathrm{C} \mathrm{y} 7 \mathrm{~V}$, pero con baja resistencia a la corrosión por lo que se desarrolló un recubrimiento alterno para adicionar una capa de cobreado alcalino obteniendo tres capas, cobre-níquel-cromo, incrementando de esta manera la resistencia a la corrosión.

Palabras claves: Cromado metálico, resistencia a la corrosión, adherencia, aros de buses, cobreado alcalino

\section{Introducción}

Actualmente existe un creciente uso del recubrimiento metálico (estrato) sobre la superficie de aros para buses, como el cromado electrolítico y su finalidad es de protección contra la corrosión y embellecimiento del metal base (sustrato). Así como también, en algunos países sudamericanos, existe un proceso de certificación obligatorio, para aquellas autopartes consideradas de seguridad que deben tener recubrimiento metálico (como amortiguadores, extremos de dirección, etc.).

La aplicación de un proceso posterior al cromado con el objeto de mejorar la resistencia a la corrosión ya se ha estudiado previamente como es el caso de la aplicación de nitruración post-oxidación iónica en muestras de acero AISI 1045, mediante estos se ha comprobado que es posible obtener características similares de dureza comparadas con el típico cromo duro reduciendo consideradamente la tasa de contaminación ambiental como un beneficio extra, (Díaz, 2010: pp. 1-6) siendo este último criterio también resaltado por otros autores (Arango, 2007: pp. 1-160). En otra investigación sobre microdureza y espesor de capas de carburos cromadas en aceros AISI 1055 se ha analizado un tratamiento térmico de temple - revenido bajo, con el que se pudo elevar significativamente la microdureza de las muestras disminuyendo así el riesgo de falla por altas tensiones y al mismo tiempo manifestando que un incremento de la rugosidad superficial favorece en el incremento del espesor de las capas nombradas. (Gonzalez, 1999: pp. 33-36).

El comportamiento de estos recubrimientos metálicos se evalúa mediante diferentes técnicas y ensayos, obteniéndose propiedades como: la adherencia (Egidi, 2012), la velocidad de corrosión (Tristancho, 2015: p.32), el desgaste, el sinergismo, la morfología y la composición del recubrimiento (Ortega, 2016: pp. 145). Se han analizado en ciertos estudios, mediante experimentación, la resistencia a la corrosión estudiando recubrimientos de cromo y zinc, finalmente se recalca que, en cuanto a los recubrimientos de 
$\mathrm{Cr}$, su proceso corrosivo se encontró dominado por la presencia de poros e imperfecciones en la capa y así también se corroboran las diferencias tanto en el rendimiento como en los mecanismos de protección en dichos recubrimientos. (Torres y Ascolani, 2011: pp. 171-185). Ciertos autores coinciden con el criterio anterior en cuanto a que la capa de cromo posee una naturaleza microfisurada (Suárez, 2003: pp. 468-471) y si esta no es lo suficientemente gruesa, el agente corrosivo podría llegar al substrato a proteger (Julve, 2001: pp. 19-27).

Al existir poca adherencia del recubrimiento metálico, se puede producir un desprendimiento progresivo de la capa de cromo durante el funcionamiento de las partes mecánicas que están en contacto, lo que puede generar varios problemas de desempeño, por lo general el material con mayor efecto sinérgico es el sustrato (Aperador, 2014: pp. 23-32), es decir que las partes con recubrimiento metálico tienen una mejor resistencia a la corrosión. La resistencia a la corrosión del recubrimiento metálico puede depender de la temperatura del electrolito utilizado (Torres y Olaya, 2013: pp. 109-125), de la deformación plástica producida por las cargas de compresión (Girardin, 2012: pp. 1-6), por el número de capas del recubrimiento, que pueden ser diferentes compuestos (Cifuentes y Olaya, 2014; Meng y Ji, 2014: pp. 1785-1790), siendo las más relevantes. Recientemente se han realizado estudios de recubrimientos metálicos sobre polímeros mediante el uso de películas nano compuestas (Ram, 2016: pp. 325-330), y del manejo de los residuos que se producen en el proceso de cromado (Alves y Seo, 2014: pp. 423-434).

En Ecuador, es muy común encontrar la superficie de los aros deteriorados, corroídos y de una mala apariencia. Se podría indicar que una de las principales causas es la incorrecta aplicación de la capa cromada a la superficie por falta de control en el proceso. Hasta el momento se ha tratado de mejorar este proceso, pero sin buenos resultados.

Las empresas locales realizan este tipo de recubrimiento utilizan dos capas $(\mathrm{Ni}-\mathrm{Cr})$, sin tener una información técnica de la incidencia de este recubrimiento sobre el metal base.

En este trabajo se plantea un estudio de la influencia de varios parámetros de cromado, como el tiempo, temperatura y voltaje, aplicado al baño de níquel durante la exposición de este elemento, seguido con las pruebas de laboratorio como las de adherencia, dureza, espesor de capa y resistencia a la corrosión con el propósito de mejorar este tipo de recubrimiento.

\section{Metodología}

Este estudio usó las técnicas de investigación bibliográfica y de campo, a nivel exploratorio, descriptivo, analítico y prospectivo. Para el 
logro de cada uno de los objetivos planteados se empleó la metodología descrita a continuación:

\section{Proceso de cromado electrolítico}

Este proceso se realizó en 15 muestras, variando el tiempo de exposición en baño de níquel de 30, 60 y 90 minutos, bajo la temperatura de trabajo de 30,35 y $40{ }^{0} \mathrm{C}$ y el de voltaje de 6,7 y $8 \mathrm{~V}$.

\section{Determinación de los aros y tipo de material}

El punto de partida para el presente estudio fue la obtención del aro, para el análisis de los mismos fueron determinados en base a la marca de buses que más ocupa el mercado nacional siendo el Hino (Anuario de estadística de transporte INEC, 2013). El material que está compuesto el aro es acero al carbono SAE- 1026, y su forma está dado por un proceso de conformado en frio.

\section{Trazado y obtención de probetas}

De acuerdo a la norma INEN 954- 7.1, el tamaño de la muestra se lo decide de acuerdo con las partes interesadas, por lo tanto se construyó las probetas con un área de $140 \mathrm{~cm}^{2}$. El seccionamiento se realizó por medio de un corte plasma, se seleccionó este método porque el calor que produce es puntual y no afecta a las propiedades del material.

\section{Preparado de probetas}

El preparado de las probetas consistió en el retiro de la escoria de su contorno y la elaboración de un agujero de un cuarto de pulgada que permitió colocar la ganchera de soporte a los baños electrolíticos.

\section{Despintado de probetas}

Por lo general los aros de los buses vienen pintados de color plomo con la finalidad de proteger contra la corrosión y dar una buena apariencia. Cuando deseamos realizar un recubrimiento metálico como el cromado, necesitamos realizar el retiro de la pintura y para este efecto lo quemamos a una temperatura de $350{ }^{\circ} \mathrm{C}$ por medio de un quemador a base de GLP (gas licuado de petróleo).

\section{Decapado de probetas}

Por el proceso de despintado y por la exposición del acero sin protección a medios ambientales, este tiende a oxidarse y a corroerse por lo que necesitamos hacer una limpieza de las superficies corroídas y para este efecto realizamos el proceso de decapado, que consiste en la inmersión de las probetas en una solución de ácido sulfúrico al 10\% con agua, por un lapso de 
una hora, pudiendo este aumentar dependiendo del grado de corrosión, posteriormente se lo realiza un neutralizado sumergiendo estas piezas en una solución de sosa caustica.

\section{Proceso de pulido}

Una vez que se obtuvo las piezas decapadas se procedió a realizar el pulido, que consistió en aplicar presión a la pieza a pulir sobre unos rodillos hechos a base de discos de tela pegados en su periferia material abrasivo, que giran a una velocidad de $3000 \mathrm{rpm}$, hasta que el elemento presente una superficie homogénea y libre de rayaduras.

\section{Desengrase electrolítico}

Para eliminar la grasa provenientes de procesos anteriores, se procedió a realizar un desengrase electrolítico, en un baño donde el electrolito fue una solución alcalina a base de detergentes, sosa caustica y carbonato de sodio, la misma que estuvo a una temperatura de $30^{\circ} \mathrm{C}$, haciendo de cátodo la pieza y de ánodo la tina, esto se llevó a efecto por un tiempo de 2 a 3 minutos con una aplicación de una tensión de $6 \mathrm{~V}$.

\section{Niquelado}

Para este efecto primero realizamos un enjuague y activado en una solución de ácido sulfúrico al $10 \%$ de concentración, luego se procedió a realizar el niquelado de las probetas utilizando una celda electrolítica. En este proceso se suspendió las probetas en las barras de agitación para luego ser sumergido en el electrolito cuyas características se observa en el cuadro 1, las mismas que en este caso hacen de cátodo. Los ánodos de níquel se colocaron en dos barras de cobre fijas en la parte lateral de la tina. Luego se accionó el sistema por un tiempo de 30 a 90 minutos y con una tensión de 6 a 8 voltios.

Cuadro 1: Características del electrolito para niquelado

\begin{tabular}{|c|c|}
\hline \multicolumn{2}{|c|}{ Características } \\
\hline $\mathrm{Ph}$ & 4 \\
\hline Densidad $\left({ }^{\circ} \mathrm{B}\right)$ & 21 \\
\hline $\mathrm{NiSO}_{4} 6 \mathrm{H}_{2} 0(\mathrm{~g} / \mathrm{l})$ & 280 \\
\hline $\mathrm{NiCI}_{2} 6 \mathrm{H}_{2} 0(\mathrm{~g} / \mathrm{l})$ & 50 \\
\hline $\mathrm{H}_{3} \mathrm{BO}_{3}(\mathrm{~g} / \mathrm{l})$ & 30 \\
\hline $\mathrm{Base}_{\left(\mathrm{cm}^{3} / \mathrm{l}\right)}$ & 40 \\
\hline Abrillantador $\left(\mathrm{cm}^{3} / \mathrm{l}\right)$ & 2.5 \\
\hline Antiporo $\left(\mathrm{cm}^{3} / \mathrm{l}\right)$ & 7 \\
\hline
\end{tabular}

Fuente: Investigación bibliográfica, 2016

Realizado por: Los autores. 


\section{Cromado}

Luego del niquelado se procedió a realizar el cromado electrolítico que no es más que el depósito de una capa fina de cromo con el fin de proteger de los ambientes corrosivos y dar una mejor apariencia, este proceso se realizó con un baño de cromo en condiciones que se muestra en el cuadro 2.

Cuadro 2: Condiciones de trabajo del baño de cromo

\begin{tabular}{|c|c|c|}
\hline \multicolumn{3}{|c|}{ Baño de Cromo Decorativo } \\
\hline Ácido Crómico & \multicolumn{2}{|c|}{$250-255 \mathrm{~g} / \mathrm{l}$} \\
\hline Densidad de Corriente & \multicolumn{2}{|c|}{$3-20 \mathrm{~A} / \mathrm{dm}^{2}$} \\
\hline Temperatura & $18-25^{\circ} \mathrm{C}$ & $32-40{ }^{\circ} \mathrm{C}$ \\
\hline Aditivo CKA & $8 \mathrm{ml} / 1$ & $15 \mathrm{ml} / 1$ \\
\hline Aditivo CRB & $30 \mathrm{ml} / 1$ & $5 \mathrm{ml} / 1$ \\
\hline Aditivo CRC & $2 \mathrm{ml} / 1$ & $4 \mathrm{ml} / 1$ \\
\hline
\end{tabular}

Fuente: Investigación bibliográfica, 2016

Realizado por: Los autores.

El tiempo de inmersión es mayor a 3 segundos dependiendo del tamaño de la pieza, a una temperatura de $30^{\circ} \mathrm{C}$ con una tensión de 6 a 7 voltios.

\section{Pruebas y ensayos}

\section{Prueba de adherencia}

La prueba de adherencia es medir la fuerza con la que el revestimiento metálico está fijado a otra superficie también metálica. Para este efecto nos basamos en la norma INEN 950 - 4.6 que es un método de la cinta adhesiva, la misma que tiene un esfuerzo de unión de 290 a $310 \mathrm{gr} / \mathrm{cm}^{2}$.

Para obtener los resultados se utilizó un equipo cortador de trama cruzada elcometer 107, que puede hacer la evaluación instantánea de la calidad de unión al sustrato, ideal para revestimientos finos, gruesos o duros sobre todas las superficies, además se utilizó una cinta adhesiva ASTM D 3359 - D.

\section{Procedimiento para medir la prueba de adherencia}

1. Presionar con fuerza y jalar hacia atrás con movimiento continuo asegurándose que atraviesa el revestimiento y llega al sustrato.

2. Colocar el filo de corte con un ángulo de $90^{\circ}$ con respecto al anterior y cortar similar al paso dos tratando de crear una trama cruzada.

3. Posteriormente limpiar con una brocha los residuos e inspeccionar que los cortes hayan atravesado.

4. Cortar un trozo de cinta ASTM de aproximadamente $75 \mathrm{~mm}$ y pegar sobre el entramado, asegurándose que este bien adherida. 
5. Transcurrido 90 segundos más o menos se retirar la cinta con movimiento suave a un ángulo de $180^{\circ}$ con respecto a la superficie del revestimiento.

6. Evaluar la adherencia comparando el entramado del corte con la norma ASTM D3359-09.

\section{Pruebas de dureza}

Para evaluar la dureza se utilizó el durómetro del laboratorio de materiales de la Universidad de la Fuerzas Armadas (ESPE) Latacunga, con una escala de dureza Vickers y bajo la norma INEN 1058.

\section{Medición de espesores}

Para obtener la lectura de los espesores se utilizó el "Medidor de espesor de pintura y recubrimiento metálicos DT-156" que es un calibrador compacto y práctico diseñado para la medición de espesores de recubrimiento no-destructivo, rápido y preciso con rango de medida $0 \sim 1250$ $\mu \mathrm{m} / 0 \sim 50$ mil. En esta fase los espesores se determinaron con la norma INEN 954 para índices de servicio 1 cuyo espesor de capa de níquel es de 10 a $12 \mu \mathrm{m}$ y la de cromo $0.3 \mu \mathrm{m}$.

\section{Pruebas de corrosión}

La prueba de corrosión se realizó bajo la norma INEN 954 para índices de servicio 1, en la que menciona que el ensayo debe hacerse de acuerdo a la norma NTE INEN 1174: 2013, para la determinación de la resistencia a la corrosión en recubrimientos metálicos, con ensayo de niebla salina acética.

\section{Resultados}

\section{Adherencia}

Para el análisis de las pruebas de adherencia se realizó un resumen de todas las pruebas hechas bajo las variables de tiempo, temperatura y tensión de trabajo como se puede observar en la tabla 3.

Cuadro 3: Medición de adherencia

\begin{tabular}{|c|c|c|c|c|}
\hline \multicolumn{5}{|c|}{ Análisis de adherencia (\%) } \\
\hline Temperatura & Tensión & \multicolumn{3}{c|}{ Tiempo } \\
\hline${ }^{\circ} \mathrm{C}$ & $\mathrm{V}$ & $30 \mathrm{~min}$ & $60 \mathrm{~min}$ & $90 \mathrm{~min}$ \\
\hline 30 & & & & 50,66 \\
\hline 30 & 6 & 40 & 72 & 53,33 \\
\hline 30 & 7 & 42,66 & 66 & 60 \\
\hline 35 & 8 & 60 & 81,33 & 60 \\
\hline 35 & 6 & 56 & 72 & 80 \\
\hline 35 & 7 & 82,66 & 90,66 & 88 \\
\hline
\end{tabular}




\begin{tabular}{|c|c|c|c|c|}
\hline 40 & 6 & 80 & 97,14 & 89,33 \\
\hline 40 & 7 & 77,33 & 84 & 73,33 \\
\hline 40 & 8 & 60 & 90,66 & 88 \\
\hline Promedio & & 62,66 & 83,53 & 71,40 \\
\hline
\end{tabular}

Fuente: Investigación bibliográfica, 2016 Realizado por: Los autores.

En el cuadro 3, se observa que el valor que corresponde al parámetro de $60 \mathrm{~min}, 40^{\circ} \mathrm{C}$ y $6 \mathrm{~V}$; es el que está enmarcado bajo la norma INEN 950 tiene un valor de $97.14 \%$ que corresponde a un esfuerzo de adherencia de $301.13 \mathrm{gr} / \mathrm{cm}^{2}$, el resto de valores no cumplen con la norma.

\section{Dureza}

La dureza se procedió a medir en la capa de níquel-cromo del recubrimiento de los aros de buses, ya que los mismos estarán en contacto con agentes externos agresivos como lodos, granillos, piedras, etc. utilizando la norma INEN 1058 cuyos valores están entre 800 y 1100 Vickers (Hv), como se observa en el cuadro 4.

Tabla 4: Resultados de mediciones de dureza

\begin{tabular}{|c|c|c|c|c|}
\hline \multicolumn{5}{|c|}{ Análisis de la dureza (Hv) } \\
\hline Temperatura & Tensión & \multicolumn{3}{|c|}{ Tiempo } \\
\hline${ }^{\circ} \mathrm{C}$ & $\mathrm{V}$ & $30 \mathrm{~min}$ & $60 \mathrm{~min}$ & $90 \mathrm{~min}$ \\
\hline 30 & 6 & 1081,46 & 1084,66 & 1075,86 \\
\hline 30 & 7 & 1084,46 & 1100,8 & 1035,53 \\
\hline 30 & 8 & 1062,8 & 1079,26 & 1079,8 \\
\hline 35 & 6 & 896,53 & 1080,06 & 1078,8 \\
\hline 35 & 7 & 917,53 & 1051,13 & 1086,86 \\
\hline 35 & 8 & 1080,86 & 1129,46 & 1222,33 \\
\hline 40 & 6 & 823,33 & 842,67 & 932,67 \\
\hline 40 & 7 & 1107,86 & 1151,33 & 1298 \\
\hline 40 & 8 & 954,46 & 836,66 & 781,26 \\
\hline Promedio & & 1001,03 & 1039,55 & 1065,67 \\
\hline
\end{tabular}

Fuente: Investigación bibliográfica, 2016

Realizado por: Los autores.

En el cuadro 4, se determina que casi todos los valores están dentro de los parámetros enmarcados bajo la norma INEN 1058 para el recubrimiento de níquel-cromo.

\section{Espesores de capa de revestimiento}

Para realizar el análisis de las pruebas de espesores se tabulan los valores en la tabla 5 , con variables de tiempo, temperatura y tensión de trabajo. 
Cuadro 5: Resumen de capa de revestimiento

\begin{tabular}{|c|c|c|c|c|}
\hline \multicolumn{5}{|c|}{ Análisis de espesores $(\mu \mathrm{m})$} \\
\hline Temperatura & Tensión & \multicolumn{3}{|c|}{ Tiempo } \\
\hline${ }^{\circ} \mathrm{C}$ & $\mathrm{V}$ & $30 \mathrm{~min}$ & $60 \mathrm{~min}$ & $90 \mathrm{~min}$ \\
\hline 30 & 6 & 4,56 & 9,45 & 14,37 \\
\hline 30 & 7 & 4,53 & 10,15 & 14,71 \\
\hline 30 & 8 & 4,96 & 9,72 & 14,88 \\
\hline 35 & 6 & 5,14 & 10,58 & 14,81 \\
\hline 35 & 7 & 5,22 & 10,89 & 15,13 \\
\hline 35 & 8 & 5,51 & 11,06 & 15,56 \\
\hline 40 & 6 & 5,46 & 11,21 & 15,48 \\
\hline 40 & 7 & 5,57 & 11,38 & 15,62 \\
\hline 40 & 8 & 5,93 & 11,6 & 15,95 \\
\hline Promedio & & 5,20 & 10,67 & 15,16 \\
\hline
\end{tabular}

Fuente: Investigación bibliográfica, 2016

Realizado por: Los autores.

Del cuadro 5, se determina, que los valores correspondientes a la columna de $60 \mathrm{~min}$, son los espesores que se encuentran dentro del rango establecido por la norma INEN 954, para un índice de trabajo 1, con excepción de los valores de 9,45 y $9.72 \mu \mathrm{m}$.

\section{Resistencia a la corrosión}

Para este efecto se utilizó cinco probetas, las mismas que fueron seleccionadas de acuerdo al criterio de mayor adherencia esto es a la que corresponde a los parámetros de $\left(60 \mathrm{~min}, 40^{\circ} \mathrm{C}\right.$ y $\left.6 \mathrm{~V}\right)$, luego de ser sometido en la cámara salina a base de ácido acético por un tiempo de ocho horas se obtuvo los resultados que se detallan en el cuadro 6.

Cuadro 6: Resultados de los ensayos de corrosión

\begin{tabular}{|c|c|c|c|c|c|c|c|c|}
\hline No. & Recubrimiento & \multicolumn{5}{|c|}{ Evaluación } & Resulta \\
do & \multicolumn{2}{|c|}{$\begin{array}{c}\text { Manchas, picaduras, rajaduras } \\
\left(\mathrm{mm}^{2}\right)\end{array}$} & Índice & \\
\hline 1 & $\begin{array}{c}\text { Níquel }+ \\
\text { cromo }\end{array}$ & $\begin{array}{c}\text { defecto } \geq \\
2,5\end{array}$ & $\mathrm{si}$ & & no & $\mathrm{x}$ & 10 & $\sqrt{ }$ \\
\hline 2 & $\begin{array}{c}\text { Níquel }+ \\
\text { cromo }\end{array}$ & $\begin{array}{c}\text { defecto } \geq \\
2,5\end{array}$ & $\mathrm{si}$ & & $\mathrm{no}$ & $\mathrm{x}$ & 10 & $\sqrt{ }$ \\
\hline 3 & $\begin{array}{c}\text { Níquel }+ \\
\text { cromo }\end{array}$ & $\begin{array}{c}\text { defecto } \geq \\
2,5\end{array}$ & $\mathrm{si}$ & $\mathrm{x}$ & no & & - & $\mathrm{x}$ \\
\hline 4 & $\begin{array}{c}\text { Níquel }+ \\
\text { cromo }\end{array}$ & $\begin{array}{c}\text { defecto } \geq \\
2,5\end{array}$ & $\mathrm{si}$ & & no & $\mathrm{x}$ & 10 & $\sqrt{ }$ \\
\hline 5 & $\begin{array}{c}\text { Níquel }+ \\
\text { cromo }\end{array}$ & $\begin{array}{c}\text { defecto } \geq \\
2,5\end{array}$ & $\mathrm{si}$ & $\mathrm{x}$ & $\mathrm{no}$ & & - & $\mathrm{x}$ \\
\hline
\end{tabular}

Fuente: Investigación bibliográfica, 2016

Realizado por: Los autores. 
Analizando el cuadro 6, evidenciamos que dos muestras presentan no conformidad (x), es decir, el área de los defectos son mayores a $2.5 \mathrm{~mm}^{2}$ por lo que estas se rechazan.

\section{Discusión}

De acuerdo a (Suarez O, 2010) en su revista "Obtención de un recubrimiento de cromo decorativo a partir de soluciones de cromo trivalente", manifiesta que el recubrimiento a base de cromo convencional (Cr VI) presento mayor resistencia a la corrosión en cámara salina que el cromo trivalente (Cr III), por lo que no son muy óptimas para alcanzar la máxima efectividad de recubrimiento como se observa en el cuadro 7.

Cuadro 7: Resultado de ensayos en cámara salina.

\begin{tabular}{|c|c|}
\hline $\mathrm{t}($ horas $)$ & Observaciones \\
\hline & Cromo VI (placa 7) \\
\hline 24 & puntos de ataque corrosivo en el $20 \%$ del área expuesta \\
\hline 48 & puntos de ataque corrosivo en el $60 \%$ del área expuesta \\
\hline 72 & puntos de ataque corrosivo en el $75 \%$ del área expuesta \\
\hline \multirow[t]{2}{*}{100} & puntos de ataque corrosivo en el $75 \%$ del área expuesta \\
\hline & Cromo VI (placa 8) \\
\hline 24 & deterioro del recubrimiento en el $20 \%$ de área expuesta \\
\hline 48 & $\begin{array}{l}\text { quince puntos de ataque corrosivo en el área interna, deterioro del } \\
\text { recubrimiento en el } 20 \% \text { del área expuesta }\end{array}$ \\
\hline 72 & $\begin{array}{l}\text { ataque corrosivo en el } 10 \% \text { del área expuesta, deterioro del } \\
\text { recubrimiento en el } 20 \% \text { del área expuesta }\end{array}$ \\
\hline \multirow[t]{2}{*}{100} & $\begin{array}{c}\text { ataque corrosivo en el } 20 \% \text { del área expuesta, deterioro del } \\
\text { recubrimiento en el } 20 \% \text { del área expuesta }\end{array}$ \\
\hline & Cromo III (placa 13) \\
\hline 24 & $\begin{array}{l}\text { ataque corrosivo en el } 60 \% \text { del área expuesta, deterioro del } \\
\text { recubrimiento en el } 90 \% \text { del área expuesta }\end{array}$ \\
\hline 48 & $\begin{array}{l}\text { ataque corrosivo en el } 60 \% \text { del área expuesta, deterioro del } \\
\text { recubrimiento en el } 90 \% \text { del área expuesta }\end{array}$ \\
\hline 72 & $\begin{array}{c}\text { ataque corrosivo en el } 70 \% \text { del área expuesta, deterioro del } \\
\text { recubrimiento en el } 90 \% \text { del área expuesta }\end{array}$ \\
\hline \multirow[t]{2}{*}{100} & $\begin{array}{l}\text { ataque corrosivo en el } 70 \% \text { del área expuesta, deterioro del } \\
\text { recubrimiento en el } 90 \% \text { del área expuesta }\end{array}$ \\
\hline & Cromo VI (placa 14) \\
\hline 24 & $\begin{array}{c}\text { ataque corrosivo en el } 40 \% \text { del área expuesta, deterioro del } \\
\text { recubrimiento en el } 80 \% \text { del área expuesta }\end{array}$ \\
\hline 48 & $\begin{array}{c}\text { ataque corrosivo en el } 80 \% \text { del área expuesta, deterioro del } \\
\text { recubrimiento en el } 90 \% \text { del área expuesta }\end{array}$ \\
\hline 72 & $\begin{array}{c}\text { ataque corrosivo en el } 80 \% \text { del área expuesta, deterioro del } \\
\text { recubrimiento en el } 90 \% \text { del área expuesta }\end{array}$ \\
\hline 100 & $\begin{array}{l}\text { ataque corrosivo en el } 80 \% \text { del área expuesta, deterioro del } \\
\text { recubrimiento en el 100\% del área expuesta }\end{array}$ \\
\hline
\end{tabular}

Fuente: Garcí, 2006. p. 6. 
Estos resultados son similares a los obtenidos en las pruebas que se desarrolló en la investigación del cromado metálico en la superficie de los aros para buses, utilizando cromado trivalente, y se demuestra con los ensayos de corrosión y adherencia; por lo que se recomienda utilizar una capa más de cobre en el proceso cuyos parámetros se muestran en el cuadro 8 , con el fin de garantizar el desempeño de este elemento en lugares de alta incidencia corrosiva, obteniendo aros cromados como se observa en la fotografía No.1.

Cuadro 8: Parámetros de recubrimiento $\mathrm{Cu}-\mathrm{Ni}-\mathrm{Cr}$

\begin{tabular}{|c|c|c|c|c|}
\hline $\begin{array}{c}\text { Tipo de } \\
\text { recubrimiento }\end{array}$ & $\begin{array}{c}\text { Espesor } \\
(\mu \mathrm{m})\end{array}$ & $\begin{array}{c}\text { Tiempo de } \\
\text { cobreado } \\
(\text { min) }\end{array}$ & $\begin{array}{c}\text { Tiempo de } \\
\text { niquelado } \\
(\text { min })\end{array}$ & $\begin{array}{c}\text { Tiempo de } \\
\text { cromado } \\
(\text { min })\end{array}$ \\
\hline $\mathrm{Cu}-\mathrm{Ni}-\mathrm{Cr}$ & $\begin{array}{c}\mathrm{Cu}=20 \\
\mathrm{Ni}=25 \\
\mathrm{Cu}=0.3\end{array}$ & 45.17 & 60 & 6.6 \\
\hline
\end{tabular}

Fuente: Investigación bibliográfica, 2016

Realizado por: Los autores.

Fotografía No. 1. Aro de bus Hino recubierto de $\mathrm{Cu}-\mathrm{Ni}-\mathrm{Cr}$

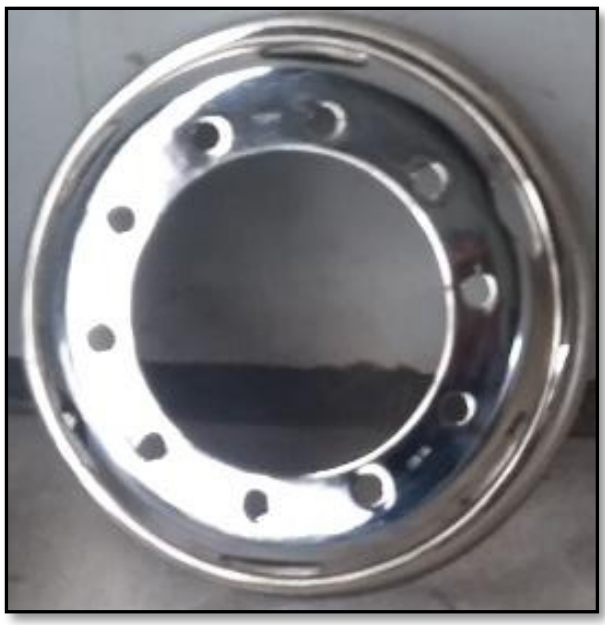

Fuente: Investigación bibliográfica, 2016

Realizado por: Los autores.

\section{Conclusiones}

En el estudio realizado de la capa cromada de los aros de buses, se pudo concluir que su adherencia al sustrato depende en gran medida de la forma en que se determine las variables del tiempo, la temperatura y la tensión eléctrica que se proporcione al baño de níquel.

En cuanto a la adherencia, luego de haber realizado algunas pruebas variando la temperatura, el tiempo y el voltaje en el proceso de niquelado se obtuvo que la mejor opción es $60 \mathrm{~min}, 40{ }^{\circ} \mathrm{C}$ y $7 \mathrm{~V}$, que tiene una adherencia de $97.14 \%$. 
En lo referente a la dureza de la capa del recubrimiento se concluye que casi todos los valores está dentro de los parámetros que exige la norma INEN 1058 para los procesos de niquelado - cromado.

En relación al espesor de la capa niquelado- cromado, luego de hacer un análisis de las medidas tomadas aplicando los diferentes cambios de variables, se concluye que los parámetros ideales para que se cumplan con la norma INEN 954 están en un tiempo de $60 \mathrm{~min}$, de 35 a $40{ }^{\circ} \mathrm{C}$ y con voltaje de 6 a $8 \mathrm{~V}$.

Con el desarrollo de la presente investigación se ha obtenido un rango ideal de las variables que intervienen en el proceso como son la temperatura, voltaje y tiempo para cumplir con estándares con lo cual se asegura una adecuada resistencia a la corrosión.

\section{References:}

1. Alves, L y Seo, E. Caracterização do resíduo sólido proveniente do processo galvânico para valoração econômica ambiental. Engenharia Sanitaria e Ambiental, vol. 19, no. 4, pp. 423-434.

2. Aperador, W. Mejía, A. y Caicedo, J. (2014). Comportamiento de corrosión-erosión en recubrimientos de TIN/ZRN depositados sobre acero AISI 4140. Información tecnológica, vol. 25, no. 1, pp. 23-32.

3. Arango, Á., Ballesteros, S., \& Casas, G. (2007). Diagnóstico y formulación del programa de producción más limpia en la industria de galvanotecnia en el proceso de cromado. Bogotá: Universidad de la Sabana.

4. Cifuentes, H y Olaya, J. (2014). Microestructura y resistencia a la corrosión de nitruros de cromo obtenidos por nitruración gaseosa al vacío de cromo electrolítico depositado sobre acero AISI H13, Revista Ingenierías Universidad de Medellín, vol. 12.

5. Díaz, J., Granda-Gutierréz, E., member, I., Campa, A., Pérez, S., Garza, A., Méndez, R. (2010). Nitruración post-oxidación iónica como técnica alternativa al cromado electrolítico. VIII Congreso Internacional sobre Innovación y Desarrollo Tecnológico, 1-6.

6. Egidi, D. Martynenko, Calahonra, C. Szombach, J y Martinez, D. (2012). Influencia del espesor de cromado en la vida útil de amortiguadores convencionales sometidos al ensayo de durabilidad. Unpublished.

7. Garci, O. (2006). Obtaining decorative chromium plating from trivalent chromium solutions, Ingenieria e investigacion, vol. $26, \mathrm{n}^{\mathrm{o}} 2$, p. 6

8. Girardin, P. Frigerio, A y Bertod, P. (2012). Modification of the Corrosion Properties of a Model Fe-8Ni-18Cr Steel Resulting from 
Plastic Deformation and Evaluated by Impedance Spectroscopy, ISRN Corrosion, vol. 2012, pp. 1-6.

9. Gonzalez, J., Quintana, R., Duffus, A., Rodriguez, A., Febles, J., \& Jimenez, M. (1999). Algunas experiencias en el incremento de la microdureza y el espesor de capas de carburos cromadas por difusión. Ingeniería Mecánica, 33-36.

10. Julve, E. (2001). Perspectiva general del cromado industrial: Características fisicas del recubrimiento y tipos de cromado. Química e Industria, 19-27.

11. Meng, J y Ji, Z. (2014). Effect of different fillers on oxidation behavior of low-temperature chromizing coating, Transactions of Nonferrous Metals Society of China, vol. 24, no. 6, pp. 1785-1790.

12. Ortega, A. Ocampo, F y Forleler, E. (2016). Comportamiento de un recubrimiento bicapa de $\mathrm{Cr} / \mathrm{CrN}$ bajo degradación por erosióncorrosión por ensayo de impacto con chorro, DYNA, vol. 83, no. 197, p. 145.

13. Ram, M. Goswami, D. Takshi, A y Stefanakos, E. (2016). Comportamiento de un recubrimiento bicapa de $\mathrm{Cr} / \mathrm{CrN}$ bajo degradación por erosión-corrosión por ensayo de impacto con chorro, DYNA, vol. 83, no. 197, p. 145.

14. Suárez, B., Zalazar, M., Salinas, D., \& Bessone, J. (2003). Fallas de recubrimientos metálicos empleados en equipos de producción de petróleo. Jornadas SAM, 468-471.

15. Torres, M., \& Ascolani, H. (2011). Resistencia a la corrosión de recubrimientos electroquímicos de cromo y zinc mediante EIE. Ingeniería y Desarrollo, 171-185.

16. Torres, M. Olaya, J. (2013). Efecto de la temperatura del electrolito sobre la resistencia a corrosión de recubrimientos de nitruro de cromo, cromo duro y pinturas tipo epoxy, Ingeniería y Universidad, vol. 17, pp. 109-125.

17. Tristancho, R. Holguín, P y Ramírez, L. (2015). Corrosión a alta temperatura de los recubrimientos metálicos $\mathrm{NiCr}$ y $\mathrm{NiCrBSiFe}$ depositados mediante proyección térmica, Prospectiva, vol. 13, no. 1, p. 32 . 